Sir,

\section{Fatal thrombotic thrombocytopenia purpura}

This is an interesting case which highlights one of the many presentations of Thrombotic thrombocytopenic purpura which also include retinal haemorrhages, oculomotor nerve palsies, pupil abnormalities as well as our case of retinal vascular occlusion and the case of choroidal vasculopathy as described by the authors. It is important to recognise this spectrum of presentations, as Thrombotic thrombocytopenic purpura can be fatal if not diagnosed and treated promptly.

\section{T Ong}

Department of Ophthalmology, Royal Free Hospital, Rowland Hill Street, Hampstead, London, NW3 2QG, UK

Correspondence: T Ong,

Tel: + 447941560168 ;

Fax: + 442086929111

E-mail: tuyen.ong@doctors.org.uk

Eye (2006) 20, 1074. doi:10.1038/sj.eye.6702103; published online 30 September 2005

Sir,

Ten year follow up of pigmentary retinopathy associated with 3-hydroxyacyl-CoA dehydrogenase deficiency

Mitochondrial $\beta$ oxidation is the principal pathway by which energy is obtained from fatty acids. Defects in this pathway are associated with hypoketotic hypoglycaemia, hepatic steatosis, rhabdomyolysis, cardiomyopathy, hypocarnitaemia, and abnormal urinary metabolites. Long-chain 3-hydroxy-acyl-CoA dehydrogenase (LCHAD) deficiency disrupts this pathway and was first described in $1989 .{ }^{1-4}$ Treatment is with a low-fat high-carbohydrate diet, carnitine and docozahexanoic acid (DHA) supplementation. Pigmentary retinopathy has been associated with this abnormality. ${ }^{5-9}$ We report such a case with follow-up over a 10 year period.

\section{Case report}

A 5-year-old emmetropic girl with a history of LCHAD deficiency was referred to the ophthalmology department for evaluation. She was on vitamin supplementation, carnitine and a low-fat highcarbohydrate diet. At first presentation, her visual acuity was recorded at 6/6 in each eye. Fundal examination was documented as showing a bilateral pigmentary retinopathy. During the first 6 years of follow-up her visual acuity remained at a similar level. At the age of 11 a myopic prescription was prescribed, right -0.75 DS / $-1.0 @ 180$, left -0.5 DS/ $-0.75 @ 180$, visual acuity remaining 6/6 in each eye. At the age of 15 the patient complained of visual deterioration. Visual acuity in the right eye had deteriorated to $6 / 24$ while the left eye retained $6 / 6$ vision. The degree of myopia had increased to right $-4.0 \mathrm{DS} /-1.75 @ 10$, left -3.25DS/-1.0@170. Ophthalmoscopy showed extensive posterior pole atrophy (Figure 1) in each eye with an 'island' of retinal pigment epithelium retained in the left eye (Figure 1b). A small hyper-pigmented spot was also noted at the fovea of each eye. The periphery of each eye showed granular RPE changes. Retinal vasculature was of normal appearance. Anterior segments were normal.
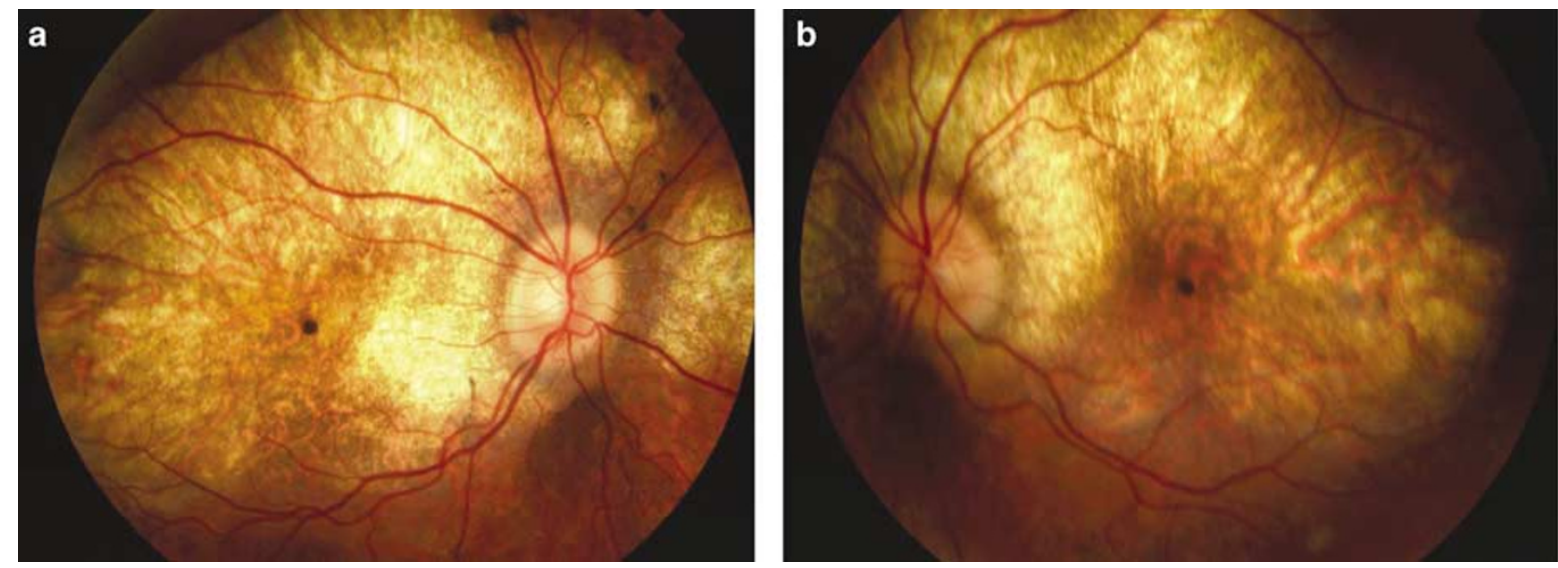

Figure 1 Endoscopic findings showing the well-demarcated atrophic posterior pole in each eye (a) right eye (b) left eye. Note the hyperpigmented spots at each macula. 